\title{
Establishing World Cultural Heritage Sites Resource Allocation System and Management System based on Neural Network Algorithm-A Case on Hailongtun Tusi Site
}

\author{
Wei Ren ${ }^{\mathrm{a}}$, Qinyun Liu ${ }^{\mathrm{b}}$, and Meiyu Shi ${ }^{\mathrm{c},}$ \\ ${ }^{a}$ UNESCO World Heritage Institute of Training and Research - Asia and Pacific (Shanghai)/Tongji University, Shanghai, 200092, China \\ ${ }^{b}$ Centre for Creative Computing, Bath Spa University, Bath, SN13 OBZ, England, UK \\ ${ }^{c}$ Tourism Institute, Beijing Union University, Beijing, 100083, China
}

\begin{abstract}
A World Heritage Site must be of outstanding universal value and meet at least one out of ten selection criteria that in some respect as a geographically and historically identifiable place having special cultural or physical significance. Therefore, an increasing number of World Heritage Sites have been developed to be the tourist attractions worldwide. Tourism resources of the World Cultural Heritage Sites are used for protecting the heritage and providing better service for the tourists. Therefore, achieving a reasonable and effective resource allocation and resource supervising system is required. This research aims at solving such problem. The artificial intelligence techniques, especially the neural network algorithm is used in this research to complete massive calculations, including facing detecting and recognising, resource elements parameters calculation, and so forth. Creative computing theories and methods are working for combining the tourism area with computer science area. To express the system more explicitly, a case about Hailongtun World Cultural Heritage Site, which located in the Guizhou province in China, is used for testing the system.
\end{abstract}

Keywords: World Cultural Heritage; neural network; creative computing; allocating and management system

(Submitted on November 18, 2018; Revised on December 15, 2018; Accepted on January 11, 2019)

(C) 2019 Totem Publisher, Inc. All rights reserved.

\section{Introduction}

A World Heritage Site is a place that is listed by the United Nations Educational, Scientific and Cultural Organization (UNESCO) as having special cultural or physical significance. To be included on the World Heritage List, sites must be of outstanding universal value and meet at least one out of ten selection criteria [1]. World Cultural Heritage Site is one of the most favorite destinations for visitors. The cultural tourism also is becoming a fastest-growing global tourism markets. Various World Heritage Sites have been developed to be the tourist attractions worldwide. However, an increasing number of visitors cause the significant issues in the most World Cultural Heritage Site, for instance, inadequate integration of cultural heritage protection and management, inequitable balance in conservation, interpretation and tourism development, excessive, poorly planned or unmonitored tourism activities. Moreover, an emerging situation that is the most World Heritage Sites have limited human resource to deal with the more visitors in developed and moderately developed countries. Therefore, such the emerging situation are forcing the world heritage sites to achieve a reasonable and effective resource allocation and resource supervising system. This research aims at establishing a system to achieve allocating the tourism resources effectively and reasonably for the World Cultural Heritage Sites. Resource allocating approach is based on heritage tourism rules and heritage protection principles to allocating in the heritage site. Reasonable and available allocation scheme can be generated through neural network. Through conventional neural network, computer can achieve pattern recognising. About the complex calculation for providing data for report generation, back propagation neural network is available. Then the entire infrastructure and resources are supervised by the system. Errors can be detected through real-time monitoring. New scheme is formulated to solve errors through the main approach for maintaining reasonable resource allocation. Creative computing theories and methods are working for combining the tourism area with

\footnotetext{
* Corresponding author.

E-mail address: jingdl@cczu.edu.cn
} 
computer science area. The whole system includes three steps, which are resource allocation function, resource supervising function and cultural heritage site supervising function. To express the system more explicitly, a case about Hailongtun World Cultural Heritage Site, which located in the Guizhou province in China, is used for testing the system.

To explain the system more explicitly, this paper is composed of five sections, which are introduction, related work and background, main approach explanation, case study about Hailongtun World Cultural Heritage Site, and conclusion.

\section{Related Work}

\subsection{Explaining Culture Heritage Resources and Relationships Between Tourists and Heritage}

The characteristics of built heritage attractions include variations in value and identity, the selective nature of heritage, image-based marketing, perishability, seasonality, inseparability and heterogeneity.

\subsubsection{Variations in Value}

The value of heritage may vary from one person to another; thus, its definition may vary according to different observers. Different forms of heritage have varying significance to individuals, organizations and communities tied to their attitudes, values, situations and the nature of the heritage. It may also be interpreted differently within any one culture at any one time, and between cultures and through time. The same heritage may assume different meanings on a variety of scales [2-3]. For instance, Potala Palace in Tibet was originally built for religious activities and still serves as a place of spiritual enlightenment for Vajrayana Buddhists. It was designated as a World Heritage Site in 1994. Although almost everyone accepts it is a heritage site, it may have varying functions and meanings for different individuals: the monks living in the palace may see it as a place for prayer, accommodation and living; the tourist visiting the palace may see it as a place for touring and enjoying the unique culture and peace of Buddhism [4]; the local residents may see it as presenting an opportunity to attract substantial numbers of tourists who can contribute to the local economy [2]. Thus, individuals may have varied opinions on the same heritage tourism site owing to the fact each person is looking at the site from a different cultural, economic and social perspective [2].

\subsubsection{Identity}

The sense of belonging to a place is the basis for identity. The heritage connotation implies quality, security and an aura of confidence [5]. It witnesses the "Past as being integral both to individual and communal representations of identity and its connotations of providing human existence with meaning, purpose and value." [5]. Some famous heritage sites draw the local residents closer together; a sense of belonging is often stimulated by local heritage. Most individuals are proud of their local heritage and believe that they may have some connection with the heritage from geography, family history or activities [5]. The heritage site may also be a place for residents to get together to hold traditional ceremonies and festivals. In some small towns, for example, although not all the individuals are religious, they may still get together in a church to have certain ceremonies which have become the traditions of local residents [6]; then the sense of belonging related to the heritage site grows among the local residents [4]. The sense of 'ownership' of heritage also exists when individuals leave their hometowns to go elsewhere; they proudly discuss the heritage in their hometown with others and treat the heritage as 'their' heritage [4]. They feel close when they meet others who share the same heritage as they do; the feeling is like meeting old friends from home.

\subsubsection{The Selective Nature of Heritage}

Built heritage is the contemporary use of the past [6-7]. Although some features may have existed for a long time, these may not be included in the general concept of heritage [6]. The function of built heritage may be like the initial aim for the buildings in the past, or it may vary.

Timothy claimed that heritage is selective and that it is kept for its social value [8]. It is selected by human beings based on their current values and meanings [8]. Buildings, material artefacts, mythologies, memories and traditions are selected and become heritage tourism resources for the present [5]. Therefore, the concept of heritage is defined by modern society based on individual demands. People require that heritage should be representative and interpretative of their culture. Hall claimed that culture is essentially concerned with the production and exchange of meaning and real, practical effects [2,9]. Heritage has both cultural and physical material, but the uniqueness of cultural identity tends to be associated more with historical stories which attract tourists to explore [2]. The cultural value of the heritage makes the physical characteristics alive and vivid [9]. 


\subsubsection{Image-based Marketing}

Although a built heritage attraction may be a living environment for the resident community, it is often treated as a consumer product with a specific image when it comes to attracting tourists. Some very attractive and picturesque built heritage attractions may simply seem old and dilapidated to the residents [10]. Thus, the image of a built heritage attraction is one of the most important factors in tourism marketing and this often provides the first mental picture, which the majority of tourists recall when the built heritage attractions are mentioned [10]. The interpretation of a built heritage attraction emerges from the image of the heritage for new visitors. In addition, the interpretation is often formulated into a representative image for promotion; this is important to attract both tourists and business investment [11-12]. Marketing relies heavily on images when preparing the marketing plan. For example, when Hawaii is mentioned, the image of a beach and the sea appear in most people's minds, whereas, when asked about Egypt, many will think of the pyramids.

\subsubsection{Perishability}

The service of heritage tourism is perishable; the service cannot be stored [13]. For most visitors, the core product of heritage tourism that they can purchase is the image of built heritage attractions and their experience of the attractions at the time [13]. The experience goes so quickly that the most common things that they bring home after a visit are images, memories and souvenirs.

\subsubsection{Seasonality}

Although certain types of heritage tourism may suffer less of a seasonal effect than other types of tourism, economic, political and other factors may also contribute to tourism demand [14]. The promotion of heritage tourism may reduce seasonal differences within the tourism industry [14].

\subsubsection{Inseparability}

The tourism industry is not like other industries which consume physical products; it cannot be separated into the process of producing and consuming. Tourism services are first sold according to tourist demand, and then they are produced and consumed simultaneously [15]. When tourists visit a built heritage attraction, the attraction produces the heritage environment; meanwhile, tourists spend the time and money needed to reach the attraction and they pay for the entrance fee or other types of consumption such as food and drink [16].

\subsubsection{Heterogeneity}

Heritage tourism tends to have variable, non-standard outputs and inconsistencies in standards and quality over time [13]. Although the experience offered by service providers may be similar, the experience of tourists may vary due to visiting different built heritage attractions on different occasions. The built heritage attractions allow all tourists to visit after paying the entrance fee. However, the service quality in built heritage attractions may be different when tourists visit the same attraction at peak and off-peak times. In addition, the weather also plays a significant role on a day out; rainy and cloudy weather may have a negative impact on tourists' mood and experience [13].

Owing to the fact that tourism services are people-based, tourism is variable; the interaction between tourists and heritage tourism providers may vary depending on personality, age and situation [13]. Service providers vary, and the same individual may not perform the same services in the same way every time. This research argues that the communication approach should be different with a child, teenagers, adults and senior citizens because they have different market requirements.

\subsection{Artificial Intelligence Algorithms}

Artificial intelligence has been well developed after big data era starts. AI has been mentioned and developed for years. The aim of developing AI is to achieve cognition by using heuristic algorithms based on human intelligence [17]. Allan Turing suggested a test to separate human and machine, which is the famous Turing test [18]. To pass the test, a computer should be programmed to provide several capabilities, which are

- Natural language process, to make the communication possible;

- Knowledge representation, to store the information; 
- Automated reasoning, to answer the questions and draw conclusions;

- Machine learning, to adapt new circumstances and detect and extrapolate patterns [17-20].

To pass the Turing test, computer vision is quite important. Conventional neural network is used for complete pattern recognition [19-20]. Traditional problems on AI research contains reasoning, knowledge, representation, planning, learning, natural language processing, perception and the capability to move and manipulate entities. To achieve these abilities by using machines, statistical methods, computational intelligence and traditional AI methods are required. Some more tools, including searching algorithms, artificial neural network, and statistics methods, probability and economics, are required to achieve artificial intelligence methods. It is a mixed subject area, including computer science, information engineering, mathematics, psychology and so forth.

Neural networks were inspired by human intelligence and human brain. Input layer, hidden layer and output layer are three basic layers in the neural network. Each of the three layers can be regarded as a neural, like the neurons in human brain.

In this research, to achieve entities detection and resource allocation, pattern recognition is necessary. Conventional neural network is applied in this system. To establish conventional neural network, several steps are required, which are reading data and data preparation, weight and bias formula preparation, defining conventional formula and pooling formula, starting the first conventional process and pooling process, starting the second conventional process and pooling process, building fully connected layer, gradient decrescent optimisation. The basic formula of conventional neural network is:

$$
h_{i j}^{k}=\tanh \left(\left(W^{k} \times x\right)_{i j}+b_{k}\right)
$$

Where $h$ represents value of each conventional neutron; $W$ represents weight value; $b$ represents bias value.

Such process is achieved in this research to complete pattern recognition in a tourism area. Arcgis software can be used to work with $\mathrm{CNN}$ algorithm to achieve testing for the heritage tourism area.

Association rules learning is a data mining algorithm. Such as Apriori algorithm, FP growth algorithm are included in this data mining set. A set of rules are formulated through training test data [21]. Frequent itemset in the target dataset can be discovered through transaction datasets [22]. Association principles can be found. Basic process of association rules learning can be explained as:

$F=\left\{i_{1}, i_{2}, i_{3}, \cdots, i_{m}\right\}$. A set of items;

Set $D$ is a transaction set; each transaction has its own ID number.

$t \subseteq F$

if $x \subseteq t$; $t$ contains $X$, which is a set of some items in $F$;

$X \subset F, Y \subset F \& X \cap Y=\varnothing$;

The confident value $\mathrm{c}$ and the support of the relationships $\mathrm{s}$ are both in $(0,1)$ range; [23]

When the transaction $D$ is given, the association rules of $X \rightarrow Y$ can be generated based on the minimum support and confidence value that the user constitutes [24].

Apriori algorithm is an essential algorithm in the association rules learning subject. The itemset can be found out in the training process. [21, 24] The itemset which is above the minimum support value, is the frequent itemset. When itemset support is less than the minimum support, related superset is not a frequent itemset, which is named downward closure property. Support of each item can be counted to select frequent itemset. Candidate itemset can be generated by using previous pass. Finally, frequent itemset is picked and sent to the next pass. This iteration can be stopped when the number of frequent itemset is constant. [21, 24]

Vector space model and spatial autocorrelation model are used for detecting relationships between different entities in the culture heritage area. Generally, vector space model aims at transferring the documents processing into vectors processing. The cos value of two different vectors can explain the similarity of two document. Spatial autocorrelation model aims at detecting spatial dependency between different entities in target area. This model is often used in geography data analysis. In geographic area, geographic data is not separated based on spatial interaction and spatial diffusion. In this research, vector space model and spatial autocorrelation model are used for exploring space between different entities in culture heritage site, which is used for generating kernel criteria for resource allocation. 


\subsection{Creative Computing Theory}

Creative computing aims at achieving interdisciplinary research or even multidisciplinary research through professional methods to generate creativity. Based on Boden's theory, creativity can be achieved through three manners, which are combinational, transformational and exploratory method [25-27]. Creative computing aims at generating creative ideas through combining different knowledge of distinct areas [28]. Conceptual blending is considered because of the intersection of two quite different frames of reference [28]. Further, conceptual combination posits that people can modify, extend or transform the knowledge they learnt in several processes [29]. Conceptual combination process can combine irrelevant ideas, concepts or other methods to be an integration [30]. Some proposed research stated that conceptual combination had a special connection with creativity [31]. Conceptual combination is an important component in existing creativity models [30]. Therefore, creativity can be generated when three elements, novel useful and surprising, are included [27]. The main tool of creative computing is knowledge combination [26]. Expert system is an application of knowledge combination. In this research, creative computing is used for combining computer science and heritage tourism area knowledge to achieve a creative system [30].

\section{World Culture Heritage Site Resource Allocation System}

Cultural heritage site tourism resource allocation and management system has three functions that can be seen in Figure 1, which are resource allocation function, resource supervising function, and resource supplementary function. The main approach is composed of conventional neural network, vector space model and spatial autocorrelation model. This section has three parts, which are explaining the functions of allocating tourism resources for the world cultural heritage sites, explaining the function of supervising allocated resources, and explaining the function of generating schemes for the resource supplementary and reasonable management.

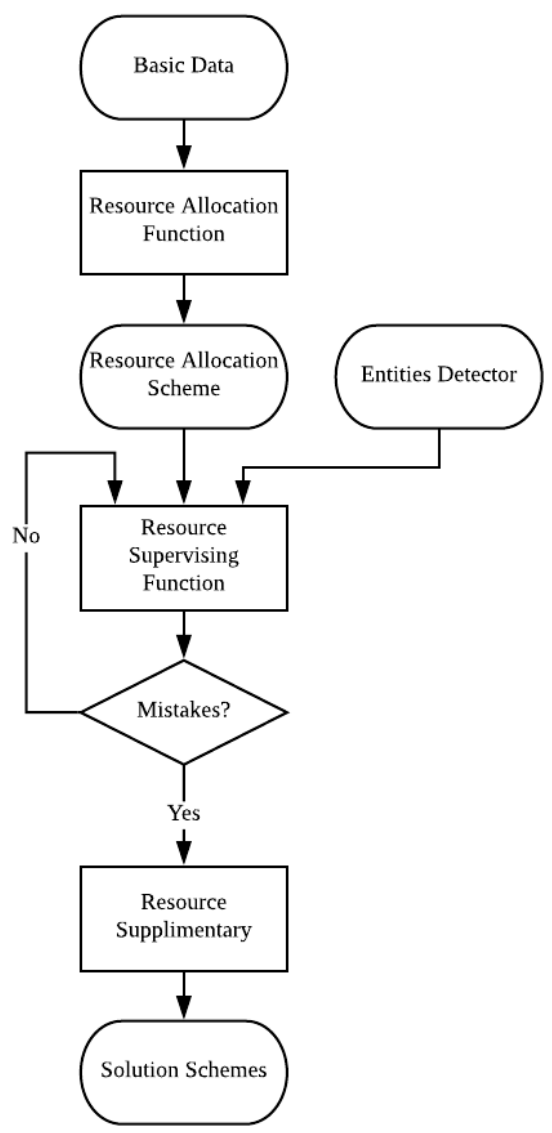

Figure 1. Cultural heritage site tourism resource allocation and management system structure

The whole system structure can be seen in the Figure 2, including three layers, which are application layer, serving layer, back-end layer. Application layer is used for providing user interface of the resources allocation and management 
system. Staff of tourism site can use the interface to manage entire site and achieve resource allocation aim. Mobile and personal computer can be operational entities. Further, some basic functions about resources allocation and management system are presented and achieved in the serving layer. Resources recognition, resources supervision, report generation, alarm function, management protocol is generated at serving layer. Relevant algorithms that are used in serving layers are also achieved at this step, including searching and matching algorithms. Searching and matching functions are important for resource recognition, which is a kernel function of serving layer. User authority management function is in the serving layer as well. In the back-end layer, cameras can collect videos and take photos for resources in the site. The media files can be stored in the servers for further use, for example analysing and reusing data to predicting tourists' motivations.

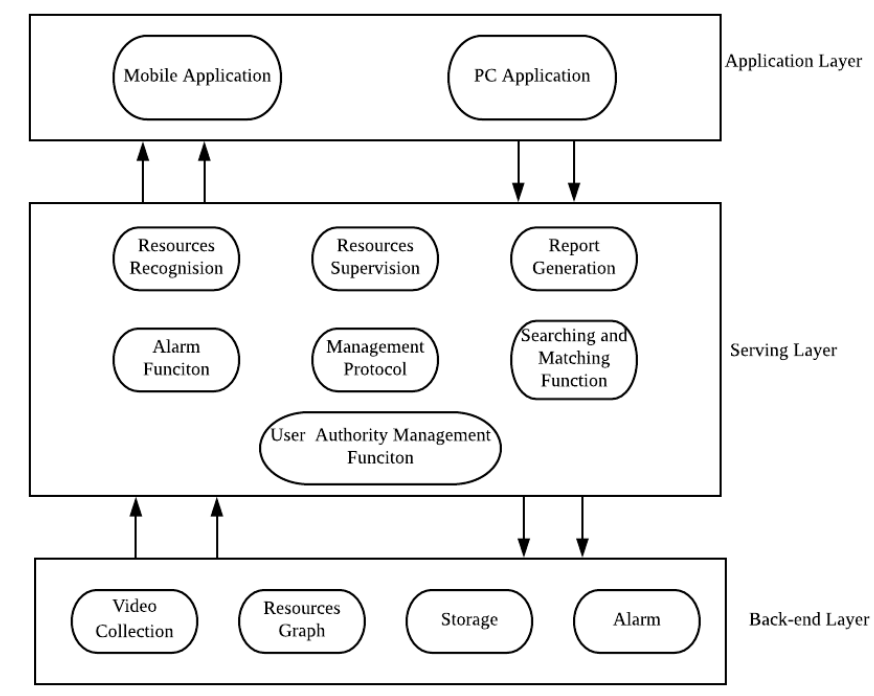

Figure 2. Resource allocation and management system architecture

The working space of this resources recognition system can be explained in the following Figure 3. The resources entities in the tourism site can be detected and being uploaded to resource recognition entities for processing and resource recognition servers for storage. Then, through the resource allocation system, user can supervise the resources situation in the tourism site and receive requirements that the system detects.

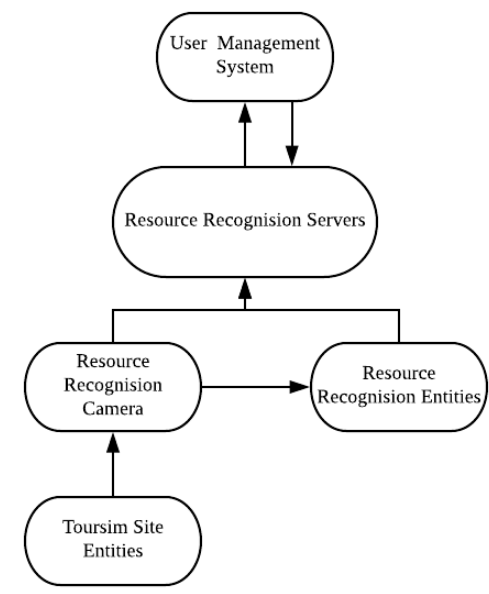

Figure 3. Working space architecture of resources allocation and management system

\subsection{Allocating Tourism Resources for the World Cultural Heritage Sites}

A world cultural heritage site usually occupies large acreage of area and is historical. Entities in the heritage area can be detected through pattern recognition algorithm. CNN algorithm is used for this step. The pictures about the heritage area are input into the system. Entities are recognised by $\mathrm{CNN}$ algorithm and classified into different categories. The detailed process is:

Input vector: 
Establishing World Cultural Heritage Sites Resource Allocation System and Management System based on Neural Network Algorithm-A Case on

\begin{tabular}{|r|r|r|r|r|r|}
\hline 15 & 190 & 168 & 234 & 23 & 12 \\
\hline 55 & 121 & 134 & 23 & 26 & 28 \\
\hline 65 & 535 & 454 & 66 & 74 & 35 \\
\hline 5 & 354 & 178 & 567 & 89 & 90 \\
\hline 11 & 788 & 587 & 267 & 5 & 32 \\
\hline 1 & 145 & 465 & 56 & 7 & 78 \\
\hline
\end{tabular}

Weight vector:

\begin{tabular}{|l|l|l|}
\hline 1 & 0 & 1 \\
\hline 0 & 1 & 0 \\
\hline 1 & 0 & 1 \\
\hline
\end{tabular}

Convolved output:

Define the size of the pattern that cognised by CNN;

The formula for the width is:

$$
W_{2}=\left(W_{1}-T+2 Q\right) / S+1
$$

The formula for the height of the picture is:

$$
H_{2}=\left(H_{1}-T+2 Q\right) / S+1
$$

Where $W_{1}$ represents the width of the pattern before conventional process; $W_{2}$ represents the width of the pattern after feature map; $H_{1}$ represents the height of the pattern before conventional process; $H_{2}$ represents the height of the pattern after feature map; $Q$ represents the padding number; $S$ represents stride; $T$ represents reception field, which is the space size of filter.

The weight vector is generated randomly. The conventional process is to possess the picture vector multiple with the weight vector. Then new conventional vectors are output. The convolved features are transferred into pooled features, which is a simple vector after pooling process. In this research, two conventional layers are built to make the pattern recognition more accurate.

The first and second conventional kernel are both $5 \times 5$. The output pictures are classified by two pooling layers. To make an optimised result, the gradient decrescent is used for discovering.

Parameters of CNN algorithm is confirmed after training. New pictures with different entities can be recognised and relevant entities can be extracted from pictures and output in categories.

Based on theories of heritage protection area and tourism area, service entities in the area should have a minimum distance between each other. For example, the dustbin in the cultural heritage area should be installed every 50 metres. Service entities are installed by Arcgis software on the heritage ichnography. The entire plan of the heritage site is stored as an essential document for protocol formulating, which are used for judging resources situations in the site.

\subsection{Supervising the Allocated Resources}

System can supervise the entire heritage tourism area after the resources have been allocated and recognised by the CNN algorithm. During tourism peak season, entities are easier being damaged by accident or on purpose by visitors. The camera in the heritage tourism area can supervise the situations. While the damaging situation happens, an alarm is triggered, which can warn staff in relevant area to focus on specific resources. Meanwhile, the system can collect entire data weekly to save in the database about the service resources to generate data report in CSV format. 


\subsection{Connection Reasoning: Knowledge Reasoning for Connection Identification}

There is a critical value about the service entities damage number (DN value). When the DN value reaches critical value, the system can output a report about re-allocating the resources and supplying resources supplementary. Missing resources can be output with specific positions after detected by supervising function. The resources names and relevant positions are the main content of report.

To make the process more explicit, Hailongtun TUSI world cultural heritage site is analysed as a case study for testing the resource allocation and management system.

\section{Case Study}

Tusi system of administrative government was aimed at unifying national administration while simultaneously allowing ethnic minorities to retain their customs and way of life. Hailongtun was established in 1257 during the Southern Song dynasty. It served as the stronghold of the Bozhou Tusi, ruled by the Yang family, from the Southern Song to the Ming dynasty. In the 28th year of the Wanli reign (1600), the Ming defeated the Bozhou rebellion, with the last Tusi Yang Yinglong committing suicide and the castle was burned down [11].

As a newly inscripted site, Hailongtun Tusi World Heritage Site faced issue between economic development and heritage protection. The tourism operation of Hailongtun by a private company, it wishes to attract more visitors, however, the World Cultural Heritage under the strict heritage protection and management that cannot host more a set tourist capacity (475 visitors) per day in the core conservation area according to the Hailongtun heritage conservation plan. It would very difficult for site management team to decide when and how allocate resources in personal when face the uncertain visitor numbers. Therefore, the artificial intelligence algorithms approach was proposed to test the possibility of tourism resources allocation. The tourism resources in this paper means numbers of guides, Security, Cleaner and bottle water.

The whole system applying in the Hailongtun tourism site can be a distributed system, including several areas subworking space to enhance the efficiency of tourism site management.

Initially, the system can detect the heritage site by recognising patterns in the pictures (Figure 4). After training through CNN algorithm, the relevant entities in Hailongtun Tusi world cultural heritage site area can be detected through pictures that captured by the camera in this tourisms site. Here is one of the paths to the peak of a mountain in Hailongtun, rocks, path, trees can be detected by the system algorithm and saved in the database. Based on analysis on this site, service resources are allocated. Such as the label for introducing this site, dustbins, protection lines for visitor's safety on two sides of the path, protection decoration for ancient wall and trees.
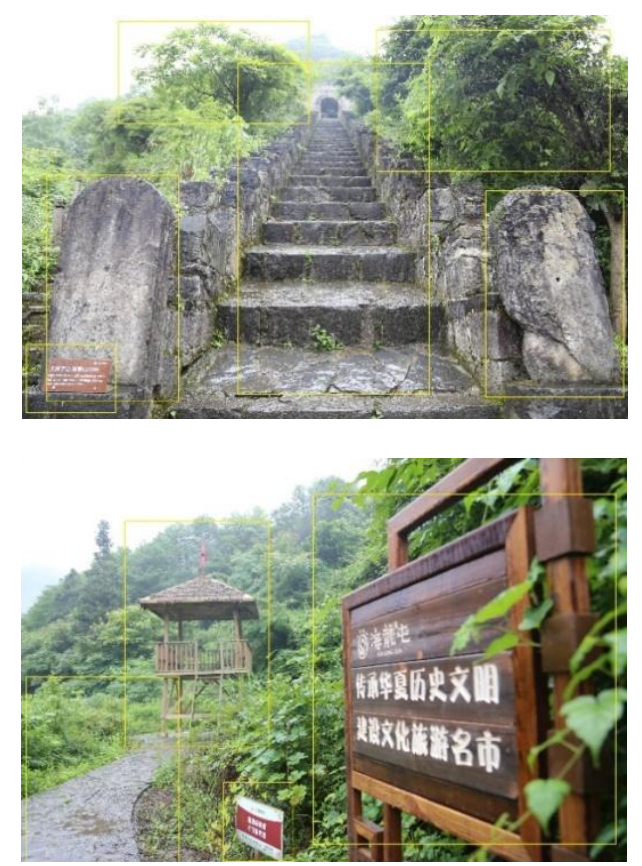

Figure 4. System pattern recognition simulation figures 
Further, the entire plan scheme of Hailongtun world cultural heritage tourism site can show the whole situation in this area. Combined with pattern recognition, the computer could recognise the plan figure, which is working on report generation. The path and service resources can be positioned (Figure 5).

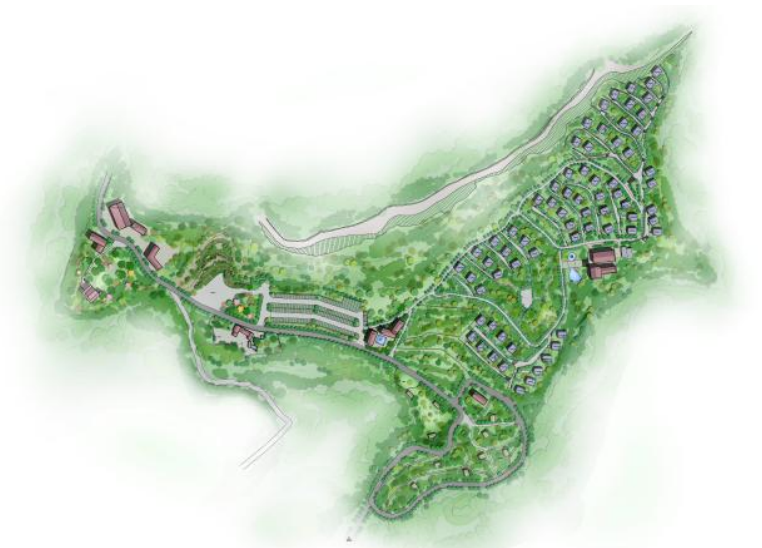

Figure 5. Hailongtun world cultural heritage tourism site entire plan scheme

In Figure 6, different function areas are presented and marked with dotted borders in different colours. Area one with blue border represents advanced camping area, restaurant, warehouse, ticket service, tourists' drop-off point, baggage storage. Area two with red border represents standard camping area. Area three with pink border represent elf-camping area and public toilets. Area four with yellow border represents tea-display area, administration area, visitor service centre, supermarket and public toilets. Area five in blue represents landscape, county restaurant, path to the mountain peak. Area six with green border represents outdoor cinema and retail stores.

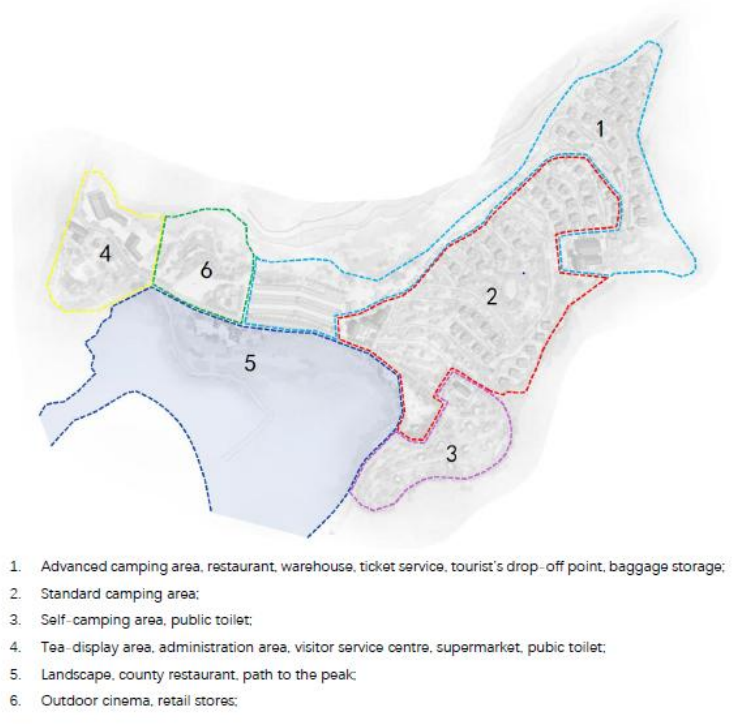

Figure 6. Hailongtun world cultural heritage tourism site function area plan

In the target area, there are several kind of service resources, which are marked in Figure 7. Tourists-serving resources, including toilets, main entrance and other entrances, exits, bicycle parking pot, car parking pot, supermarkets, cinemas, restaurants, dustbins, and telephone, are marked in the figure and can be detected by computers on the figure. After cameras catch the entities through the system, it can be connected with the position in the area through marching with the entire plan scheme.

Moreover, the label resources are detected in the same manner. The map with marks is achieved by Arcgis software, which are presented in Figure 8. Labels are function entities for guiding tourists in the tourism site. Hailongtun world 
cultural heritage tourism site is located in the mountain area, which is a complicated place. Further, tourists are unfamiliar for the new developed tourism site. Guidance is, thus, quite essential for maintaining an excellent touring and safety for all the tourists.

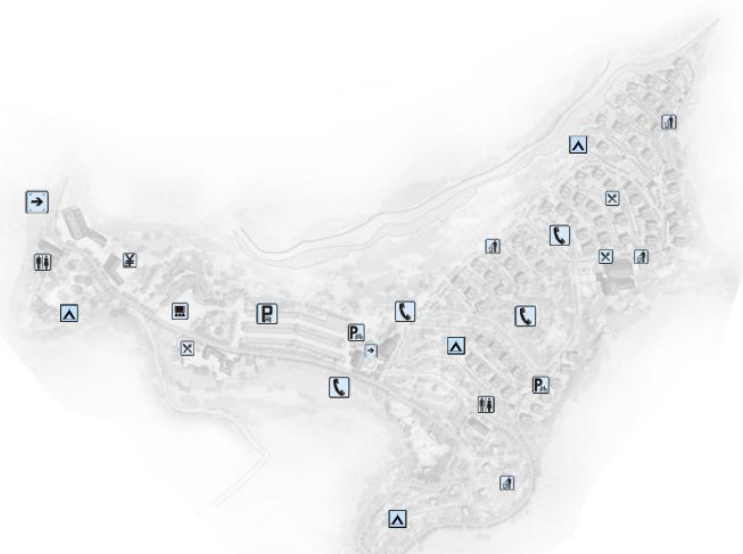

Figure 7. Service resources in Hailongtun world cultural heritage tourism site

Figure 8. Label resources in Hailongtun world cultural heritage tourism site

The lights resources can be in the Figure 9. Different colour points represent different functional light. The yellow points represent lights for the paths and main sites. The green points represent lights on the lawn. The red points represent lights for landscape, which are used for decorating tourism site to be attractive.

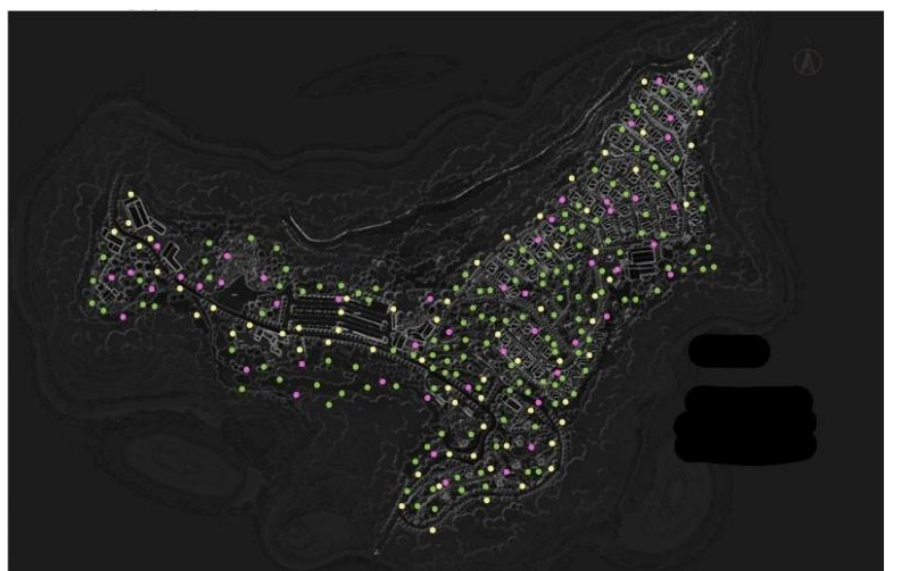

Figure 9. Light resources in Hailongtun world cultural heritage tourism site 
As the Hailongtun world cultural heritage tourism site are in the mountain area, flood protection resources should be built to protect the heritage and tourism in this area, which is presented in Figure 10.

Figure 10. Flood protection resources in Hailongtun world cultural heritage tourism site

Existing labels of Hailongtun Tusi world cultural heritage site, which are important tourism service resources for the site, can be detected as well. The system can analyse existing resources and missing resources to re-allocate the resources.

Hardware and software for pattern recognition are both necessary for this system applying in Hailongtun Tusi world cultural heritage site.

After confirming that the service resources are required supplementary, the system can output a report based on resources names, positions and numbers.

\section{Conclusions}

For world cultural heritage site, resource allocation and supervision are necessary and important for protection and tourism aspect. AI algorithm, especially the conventional neural network algorithm is effective and useful for pattern cognition. The world cultural heritage site tourism resources allocation and supervision system includes three functions to achieve these pattern recognitions, which are resource allocation function, resource supervision function and resource supplementary function. The heritage site can be protected by supervision function and service resources can be allocated reasonably in AI manner.

Further, this method can be researched applying to other areas to make resource allocation more intelligent.

\section{Acknowledgements}

This work was supported by Open Projects Fund of Key Laboratory of Ecology and Energy-saving Study of Dense Habitat (Tongji University), Ministry of Education. No. 201820302. This work was supported in part by Natural Science Foundation of China (Grant no. 71473018)

\section{References}

1. UNESCO, “The Criteria for Selection,” (https://whc.unesco.org/en/criteria/, 2018)

2. S. Hall, "Cultural Representations and Signifying Practices," Sage Publications Ltd., US, 1997

3. S. Millar, "Heritage Management for Heritage Tourism," Tourism Management, Vol. 10, No. 1, pp. 9-14, 1989

4. S. Hannabuss, "The Ashgate Research Companion to Heritage and Identity," Journal of Sustainable Tourism, Vol. 21, No. 8, pp. $1244-1245,2008$

5. B. Graham, "Heritage as Knowledge: Capital or Culture?" Urban Studies, Vol. 39, No. 39, pp. 1003-1017, 2016

6. G. Ashworth and J. Tunbridge, "Old Cities, New Pasts: Heritage Planning in Selected Cities of Central Europe," Geojournal, Vol. 49, No. 1, pp. 105-116, 1999 
7. A. Turtureanu, "Tourism Products Characteristics and Forms," Acta Universitatis Danubius Oeconomica, Vol. 1, No.1, pp. 141157,2005

8. P. Mason, “Tourism Impacts, Planning and Management," Butterworth-Heinemann, UK, 2003

9. C. Hall, "Tourism Planning: Policies, Processes and Relationships," Prentice Hall, Pearson Education Limited, Essex, England, 2007

10. A. Orbasli, "Tourists in Historic Towns," E \& FN Spon, Taylor and Francis, London, UK, 2000

11. J. Chen and X. Guo-Hong, "The Historical Geography Study on the Placename of"Hai Long Tun," Journal of Zunyi Normal College, 2012

12. H. Fisher and J. Fladmark, "The Image of A Region: The Need for A Clear Focus," The Robert Gordon University Heritage Convention, 1994

13. A. Leask and Yeoman, "Heritage Visitor Attractions: An Operations Management Perspective," Cassell, Continuum, UK, 1999

14. A. Lew, C. Hall, and A. Williams, "A Companion to Tourism," Blackwell Publishing Limited, Oxford, UK, 2004

15. D. Harrison, "Tourism and the less developed countries," Tourism and The Less Developed Countries (October), Belhaven Press, London, UK, 1992

16. J. Holloway, "The Business of Tourism," Business of Tourism, Vol. 30, No. 3, pp. 756-758, 1994

17. P. Heermann and K. Nahid, "Classification of Multispectral Remote Sensing Data using a Back-Propagation Neural Network," IEEE Transactions on Geoscience and Remote Sensing, Vol. 30, No. 1, pp. 81-88, 1992

18. T. Lee, "Back-Propagation Neural Network for Long-Term Tidal Predictions," Ocean Engineering, Vol. 31, No. 2, pp. 225-238, 2004

19. S. Haykin, “A Comprehensive Foundation,” IEEE Neural Networks, Vol. 2, No.1, pp. 41, 2004

20. M. Hagan, B. Howard, and H. Mark, "Neural Network Design," Boston PWS Publishing, Boston, USA, Vol. 20, 1996

21. X. Wu, V. Kumar, J. R. Quinlan, J. Ghosh, Q. Yang, H. Motoda, et al., "Top 10 Algorithms in Data Mining," Knowledge and Information Systems, Vol. 14, No. 1, pp. 1-37, 2008

22. M. Al-Maolegi and A. Bassam, "An Improved Apriori Algorithm for Association Rules," ArXiv.org., Cornell University Library, USA, 2014

23. J. Dongre, L. Gend, and S. V. Tokekar, "The Role of Apriori Algorithm for Finding the Association Rules in Data Mining," in Proceedings of IEEE International Conference on Issues and Challenges in Intelligent Computing Techniques (ICICT), India, 2014

24. W. Schoenmakers and G. Duysters, “The Technological Origins of Radical Inventions,” Research Policy, Vol. 39, pp. 10511059,2010

25. L. Zhang and H. Yang, "Definition, Research Scope and Challenges of Creative Computing," in Proceedings of the 19th IEEE International Conference on Automation and Computing (ICAC), pp. 1-6, London, UK, 2013

26. A. Hugill and H. Yang, "The Creative Turn: New Challenges for Computing," International Journal of Creative Computing, Vol. 1, No. 1, pp. 4-19, 2013

27. W. Schoenmakers and G. Duysters, “The Technological Origins of Radical Inventions," Research Policy, Vol. 39, pp. 10511059,2010

28. H. Yang, D. Jing, and L. Zhang, "Creative Computing: An Approach to Knowledge Combination for Creativity?" in Proceedings of IEEE Symposium on Service-Oriented System Engineering (SOSE), pp. 407-414, London, UK, 2016

29. L. Zou, Q. Liu, C. Zhang, and H. Yang, "An Approach to Applying Creative Computing in Tourism by Constructing a Big Data based Knowledge System Framework," in Proceedings of the 22nd IEEE International Conference on Automation and Computing (ICAC), pp. 244-249, London, UK, 2016

30. L. Zhang and H. Yang, "Knowledge Discovery in Creative Computing for Creative Tasks," in Proceedings of the first Conference on Creativity in Intelligent Technologies and Data Science (CIT\&DS), Vol. 535, pp. 93, Springer, London, UK, 2015

31. D. Jing and H. Yang, "Creative Computing for Bespoke Ideation," in Proceedings of the 39th IEEE Computer Software and Applications Conference (COMPSAC), Vol. 1, pp. 34-43, Taichung, Taiwan, China, 2015 